\title{
Impact of acetylsalicylic acid on tumor angiogenesis and lymphangiogenesis through inhibition of VEGF signaling in a murine sarcoma model
}

\author{
XIAOYUE ZHANG ${ }^{1,2}$, ZHAOPENG WANG ${ }^{1}$, ZHAOXIA WANG $^{1}$, \\ YUEYING ZHANG ${ }^{1}$, QING JIA ${ }^{1}$, LICUN WU ${ }^{3}$ and WEIDONG ZHANG ${ }^{1}$
}

\begin{abstract}
${ }^{1}$ Key Laboratory for Modern Medicine and Technology of Shandong Province, Institute of Basic Medicine, Shandong Academy of Medical Sciences, Jinan 250062; ${ }^{2}$ School of Medicine and Life Sciences, University of Jinan-Shandong Academy of Medical Sciences, Jinan 250062, P.R. China; ${ }^{3}$ Lanter Thoracic Surgery Research Laboratories and Division of Thoracic Surgery, Toronto General Hospital, University Health Network, University of Toronto, Toronto, Ontario, Canada
\end{abstract}

Received November 19, 2012; Accepted January 14, 2013

DOI: 10.3892/or.2013.2339

\begin{abstract}
Aspirin is a salicylate drug that is widely used, and recently it has been shown to influence the development of various types of cancers. Our previous study revealed that aspirin had an inhibitory effect on the growth of S180 sarcoma and $3 \mathrm{AO}$ human ovarian cancer cells. The present study utilized a murine S180 sarcoma model to investigate the molecular mechanisms involved in aspirin-induced tumor growth inhibition. Tumor-bearing mice were randomly divided into five groups with 10 mice in each group: i) control; ii) 5-fluorouracil (5-FU); iii) high-dose aspirin $(250 \mathrm{mg} / \mathrm{kg}$ ); iv) low-dose aspirin $(50 \mathrm{mg} / \mathrm{kg})$; and v) combination of $5-\mathrm{FU}$ and aspirin $(50 \mathrm{mg} / \mathrm{kg})$. The effect of aspirin on tumor growth was observed by measuring tumor volume and evaluating the antitumor effect. Tumor histology and immunohistochemistry were performed to detect the microvessel density (MVD), lymphatic vessel density (LVD), and the expression levels of vascular endothelial growth factor A (VEGF-A) and VEGF-C. The expression of VEGF-A and VEGF-C was also confirmed and quantified by western blotting. We discovered significant growth delay in murine S180 sarcoma as a result of aspirin treatment. The inhibition rate of tumor growth induced by highdose and low-dose aspirin was 33.5 and $22.2 \%$, respectively
\end{abstract}

Correspondence to: Professor Weidong Zhang, Key Laboratory for Modern Medicine and Technology of Shandong Province, Institute of Basic Medicine, Shandong Academy of Medical Sciences, 18877 Jing Shi Road, Jinan 250062, P.R. China

E-mail: zhangweidongkui@163.com

Professor Licun Wu, Lanter Thoracic Surgery Research Laboratories and Division of Thoracic Surgery, Toronto General Hospital, University Health Network, University of Toronto, Toronto, Ontario, Canada

E-mail: wulicun@hotmail.com

Key words: aspirin, angiogenesis, lymphangiogenesis, microvessel density, vascular endothelial growth factor
$(\mathrm{P}<0.05)$. The expression of VEGF-A and VEGF-C in tumor tissues inhibited by aspirin was demonstrated by immunohistochemistry, and the MVD was decreased in a dose-dependent manner $(\mathrm{P}<0.05)$. Reduced LVD was particularly apparent in the high-dose aspirin group $(\mathrm{P}<0.05)$. Western blot data showed that the expression of both VEGF-A and VEGF-C was reduced after treatment with aspirin. In conclusion, the impact of aspirin-induced tumor growth delay of murine S180 sarcoma may correlate with the inhibition of angiogenesis and lymphangiogenesis by reducing VEGF-A and VEGF-C expression in tumor tissues.

\section{Introduction}

The majority of cancer patients succumb to disease as a result of invasion and metastasis, since a malignant tumor is incurable and life-threatening (1). Although chemotherapy is a modality in cancer treatment, drug resistance and systemic toxicity remain major problems. Therefore, it is important and urgent to develop safe and effective antitumor drugs. While the mechanisms have not been fully elucidated (1), epidemiological and laboratory data suggest that non-steroidal anti-inflammatory agents (NSAID) have antitumor effects. Aspirin, a safe, effective and extensively used NSAID has antipyretic, analgesic, anti-thrombotic and anti-inflammatory effects through the inhibition of the activity of cyclooxygenase (COX) enzymes $(2,3)$. In recent years, aspirin was found to have potential in preventing and treating tumors, which is a major area of interest in cancer research (4). Epidemiological and clinical studies suggest that by using aspirin as a chemopreventive agent, it can reduce cancer risk by as much as $50 \%$ in some cancer types (5), including colorectal, ovarian epithelial, esophageal, bladder, lung and breast cancer as well as glioblastoma (6-12) while showing enhancement of the body's antitumor immunity (13).

The molecular mechanisms of aspirin-mediated inhibition of tumors remain unclear. Aspirin is a prototypic inhibitor of COX. Overexpression of COX-2 induced by inflammatory and mitogenic stimuli is commonly found in a variety 
of cancers (14), which suggests that COX-2 contributes to the process of carcinogenesis. Several mechanisms by which COX-2 contributes to the progression of cancer have been reported, including stimulation of proliferation and inhibition of apoptosis of cancer cells, stimulation of cancer cell invasion and angiogenesis, and suppression of immune reponses $(14,15)$. Our previous study revealed that aspirin caused an inhibitory effect on S180 sarcoma and 3AO human ovarian cancer cell growth. We discovered that aspirin decreased the expression of COX-2 in tumor tissues, and there was a positive correlation between the expression of COX-2 and angiogenic factors. Considerable evidence has shown that tumor growth and metastasis are dependent on angiogenesis, and VEGF-A is known to be one of the most important angiogenic factors. Antiangiogenesis may be one of the mechanisms by which aspirin exerts its tumor chemopreventive and therapeutic effects.

Lymph node metastasis occurs commonly in cancer with lymphatic vessels being a main channel for the spread of cancer cells $(16,17)$. Recent studies suggest that lymphangiogenesis actively contributes to metastasis based on the observations that lymphatic vessel density is correlated with the extent of lymph node metastasis $(17,18)$. Moreover, it has been revealed in some animal tumor models that the expression of lymphangiogenic growth factors leads to the formation of lymphatic vessels and that lymphangiogenesis is accompanied by enhanced lymphatic metastasis (17). Prominent expression of VEGF-C has been observed in numerous types of human cancers $(17,18)$. Several studies have shown that levels of VEGF-C expression in primary tumor were correlated with lymphatic vessel invasion (17-19). Correlations between COX-2 expression and lymphagiogenesis have been reported in several human cancer types (20-24). The present study focused on tumor angiogenesis and lymphangiogenesis by evaluating the MVD, LVD and the expression of VEGF-A and VEGF-C in tumor tissues in order to investigate the molecular mechanisms through which aspirin inhibits tumor growth.

\section{Materials and methods}

Tumor models. Fifty male Kunming mice aged from 6 to 8 weeks and weighing from 20 to $24 \mathrm{~g}$ were obtained from the Animal Experiment Center of Shandong University, China. The mouse model was provided by the Institute of Medicine, Shandong Academy of Medical Science, China. Seven days following S180 cell injection, ascites was extracted from S180 ascites sarcoma mice under an septic condition. Normal saline was then added to adjust the tumor cell concentration to $1 \times 10^{7} /$ $\mathrm{ml}$. Tumors were generated in 40 male Kunming mice by injection of a $0.2-\mathrm{ml}$ tumor cell suspension subcutaneously into the right flank of each mouse. Tap water and food were provided ad libitum.

Drugs and reagents. Aspirin was obtained from Bayer S.p.A. (Milano, Italy). DMSO was obtained from Sigma (St. Louis, MO, USA) to dissolve the aspirin. 5-FU was obtained from Tianjin Jinyao Amino Acids Co., Ltd., Tianjin, China. Western blotting related reagents were purchased from the Shanghai Beyotime Institute of Biotechnology, China.
Treatment. All tumor-bearing mice were randomly divided into 5 groups: control group, 5-FU group, high-dose aspirin group, low-dose aspirin group and combination group with 10 mice in each group. Treatment was initiated when the diameter of tumors was $5 \mathrm{~mm}$ (referred to as day 0 ) at day 5 following tumor cell injection. The high-dose and low-dose aspirin groups respectively received 250 and $50 \mathrm{mg} / \mathrm{kg}$ aspirin by oral gavage once a day for 14 days. Control mice received an equal volume of normal saline. $5-\mathrm{FU}(20 \mathrm{mg} / \mathrm{kg})$ was injected i.p. once every 3 days (day 1, 4, 7, 11 and 14). Combination group mice received $50 \mathrm{mg} / \mathrm{kg}$ aspirin by oral gavage once every day and $20 \mathrm{mg} / \mathrm{kg} 5$-FU was injected i.p. once every 3 days (day 1, $4,7,11$ and 14). The volume was measured to be $0.1 \mathrm{ml} / 10 \mathrm{~g}$. This experiment was repeated three times. The two maximal perpendicular diameters of the tumors were measured every 2 days to document tumor growth (day 0, 2, 4, 6, 8, 10, 12 and 14). Tumor measurements were converted to tumor volume (V) using the formula $\left(\mathrm{V}=\mathrm{W}^{2} \mathrm{x} \mathrm{L} / 2\right)$; where $\mathrm{W}$ and $\mathrm{L}$ are the perpendicular smaller and large tumor diameters, respectively, and plotted against time. Body weight of the mice was measured twice weekly. Mice were euthanized $24 \mathrm{~h}$ after the last treatment. The tumor masses were excised from the mice and were then weighed. Calculation of the tumor inhibitory rate was calculated using the formula: $\%$ Inhibitory rate (IR) = [average tumor weight of the control group $(\mathrm{g})$ - average tumor weight of the treatment group $(\mathrm{g})$ ]/average tumor weight of the control group (g) x 100.

Histology and immunohistochemistry. Part of the fresh tumor specimens was fixed in $10 \%$ neutral-buffered formalin, processed and embedded into paraffin blocks. Paraffin-embedded tumor samples were processed into tissue array blocks, which were cut into $4-\mu \mathrm{m}$ sections for hematoxylin and eosin (H\&E) and immunohistochemical staining. Paraffin sections were dewaxed in five changes of xylene and rehydrated through descending concentrations of alcohol. Endogenous peroxidase activities were blocked using 3\% hydrogen peroxide. Sections were treated with heating in $10 \mathrm{mmol} / \mathrm{l}$ citrate buffer at $\mathrm{pH} 6.0$ inside a water bath. They were incubated overnight in a moist chamber with the primary antibody. The secondary antibodies, biotinylated goat anti-rabbit and anti-mouse IgG (Beijing Biosynthesis Biotechnology Co., Ltd., Beijing, China), were applied at 1:200 in PBS for $1 \mathrm{~h}$ at room temperature, stained with DAB and then counterstained with hematoxylin. Finally, sections were dehydrated through graded alcohols, cleared in xylene and mounted in permount. The following antibodies were used for immunohistochemical staining: D2-40 (1:75 dilution; CWBIO, Beijing, China); CD34, VEGF-A, VEGF-C (1:100 dilution; Beijing Biosynthesis Biotechnology Co., Ltd.). Microvessel density (MVD) was analyzed in CD34stained vascular endothelial cells. CD34 is expressed in the endothelial cells of microvessels. The microvessel count was carried out in accordance with the method of Weidner et al (25). Initially, we selected 3 dense microvessel fields separately at the original magnification $\mathrm{x} 40$ and $\mathrm{x} 100$, and the numbers of CD34-stained cells were then counted at the original magnification $\mathrm{x} 400$ and averaged for statistical analysis. The method of lymphatic vessel count was identical to that of the microvessels. 
Table I. Inhibitory effect of aspirin on S180 sarcoma (mean \pm SEM, n=10).

\begin{tabular}{|c|c|c|c|c|}
\hline \multirow[b]{2}{*}{ Group } & \multicolumn{2}{|c|}{ Body weight $(\mathrm{g})$} & \multirow[b]{2}{*}{ Tumor weight $(\mathrm{g})$} & \multirow[b]{2}{*}{$\operatorname{IR}(\%)$} \\
\hline & Before experiment & After experiment & & \\
\hline Control & $24.9 \pm 2.5$ & $27.9 \pm 4.6$ & $2.84 \pm 0.71$ & - \\
\hline $5-\mathrm{FU}$ & $23.0 \pm 2.6$ & $26.3 \pm 3.0$ & $1.02 \pm 0.53^{\mathrm{b}}$ & 64.1 \\
\hline High-dose aspirin & $23.7 \pm 3.1$ & $28.3 \pm 2.3$ & $1.89 \pm 0.58^{\mathrm{a}}$ & 33.5 \\
\hline Low-dose aspirin & $24.3 \pm 4.0$ & $29.2 \pm 3.9$ & $2.21 \pm 0.89^{\mathrm{a}}$ & 22.2 \\
\hline Combination group (5-FU and aspirin) & $23.8 \pm 3.0$ & $28.5 \pm 1.6$ & $0.85 \pm 0.27^{\mathrm{b}}$ & 70.1 \\
\hline
\end{tabular}

Control, treated with normal saline. IR, inhibitory rate. ${ }^{\mathrm{a}} \mathrm{P}<0.05,{ }^{\mathrm{b}} \mathrm{P}<0.01$, vs. control.

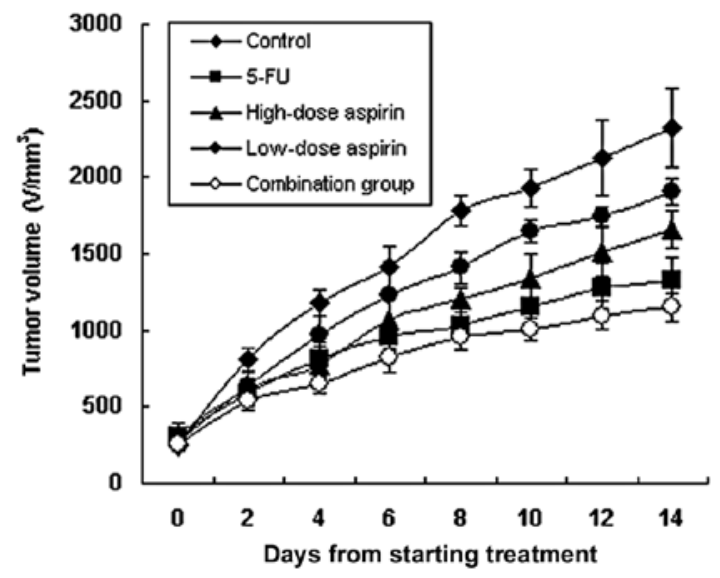

Figure 1. Growth curves of S180 sarcoma tumors after treatment. Control, treated with normal saline.

Western blotting. Sections of fresh tumor specimens were stored at $-70^{\circ} \mathrm{C}$. They were homogenized with ice-cold lysis buffer, and were then centrifuged at 10,000 rpm for $10 \mathrm{~min}$ at $4^{\circ} \mathrm{C}$. The protein concentration was determined with the BCA kit. The total protein sample was denaturated at $100^{\circ} \mathrm{C}$ for 10 min and separated by $10 \%$ SDS-PAGE gels and later transferred to Protran nitrocellulose membranes. The membranes were blocked by blocking fluid for $2 \mathrm{~h}$ and incubated overnight with an anti-VEGF-A or VEGF-C antibody (1:100 dilution), after extensive washing, and a biotinylated goat anti-rabbit IgG antibody (1:200 dilution) for $1.5 \mathrm{~h}$ at room temperature, and then stained with DAB. The band intensities were analyzed by ImageJ software (Wayne Rasband National Institutes of Health, Bethesda, MD, USA).

Statistical analysis. All data were processed by SPSS11.5 and presented as means \pm SEM. The t-test was performed to compare means and distributions for different treatment groups. Statistical significance was based on two-tail $\mathrm{P}<0.05$.

\section{Results}

Effects of aspirin on sarcoma 180 tumor growth. Tumors grew relatively rapidly in the mice. Treatment with 5 -FU $(20 \mathrm{mg}$ / $\mathrm{kg})$ alone, either high-dose $(250 \mathrm{mg} / \mathrm{kg})$ or low-dose $(50 \mathrm{mg} /$ $\mathrm{kg})$ aspirin alone, and a combination of $5-\mathrm{FU}(20 \mathrm{mg} / \mathrm{kg})$ and aspirin $(50 \mathrm{mg} / \mathrm{kg})$ resulted in inhibition of tumor growth compared to the control group. The inhibitory rate was 64.1, 33.5, 22.2 and 70.1\%, respectively (Table I, Fig. 1; $\mathrm{P}<0.05$ for each comparison). The inhibitory effect was stronger in both the combination and 5-FU groups $(\mathrm{P}<0.01)$. Although the inhibitory rates of the high- and low-dose aspirin groups were lower, the mice in both groups were in a good condition with an increased body weight after the experiment (Table I). This demonstrated that aspirin not only inhibited the growth of S180 sarcoma, but also reduced the body wasting effect caused by the tumor.

Pathological and morphometric analysis of sarcoma 180 tumors after treatment. H\&E staining showed that S180 sarcoma cells were distributed as sheets and nests. Tumor cells varied in size and shape. High- and low-dose aspirin, and the 5-FU and combination groups exhibited morphological changes characteristic of apoptotic tumor cells such as nuclear pyknosis and karyorrhexis. Microvessel density was significantly lower than that in the control group (Fig. 2). In the high-dose aspirin, 5-FU and combination groups the tumors exhibited large patchy necrosis along with the frequent observation of monocyte infiltration.

Expression of VEGF-A and VEGF-C in sarcoma 180 tumors. Based on immunohistochemical staining, VEGF-A and VEGF-C were expressed in the cytoplasm of tumor cells. Cells positive for VEGF-A and VEGF-C were stained brown (Fig. 3). The expression of VEGF-A and VEGF-C in the control group was markedly higher than that in the other treatment groups. Grey scale intensity variants of VEGF-A and VEGF-C immunoreactivity were evaluated by Leica Qwin V3 software. Sections were evaluated in each of 5 randomly selected positive regions at the original magnification $\mathrm{x} 200$. Fig. 4 indicates an inverse relationship between the grey scale intensity and the protein expression. Higher grey scale intensity indicates weaker protein expression, and lower intensity indicates stronger protein expression. Treatment with 5-FU and aspirin resulted in a reduction in VEGF-A and VEGF-C expression. VEGF-A and VEGF-C expression decreased significantly in both the 5-FU alone and combination groups when compared with the other treatment groups showing a dose-dependency on high- and low-dose aspirin. In each comparison, there was a significant difference $(\mathrm{P}<0.05)$. 


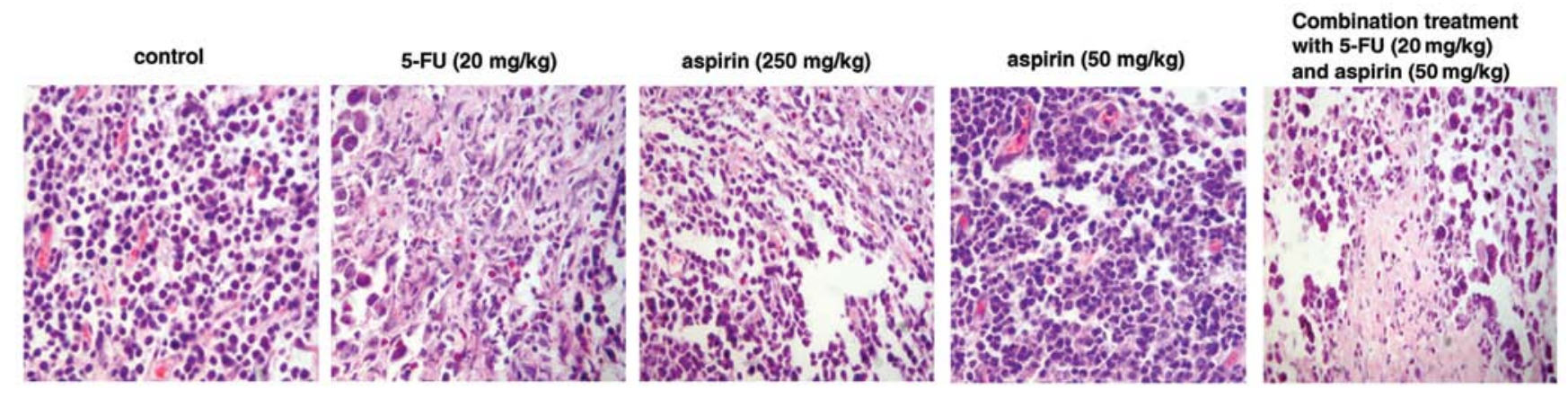

Figure 2. Pathological and morphometric analysis of S180 sarcoma tissue after treatment. Original magnification, x400.

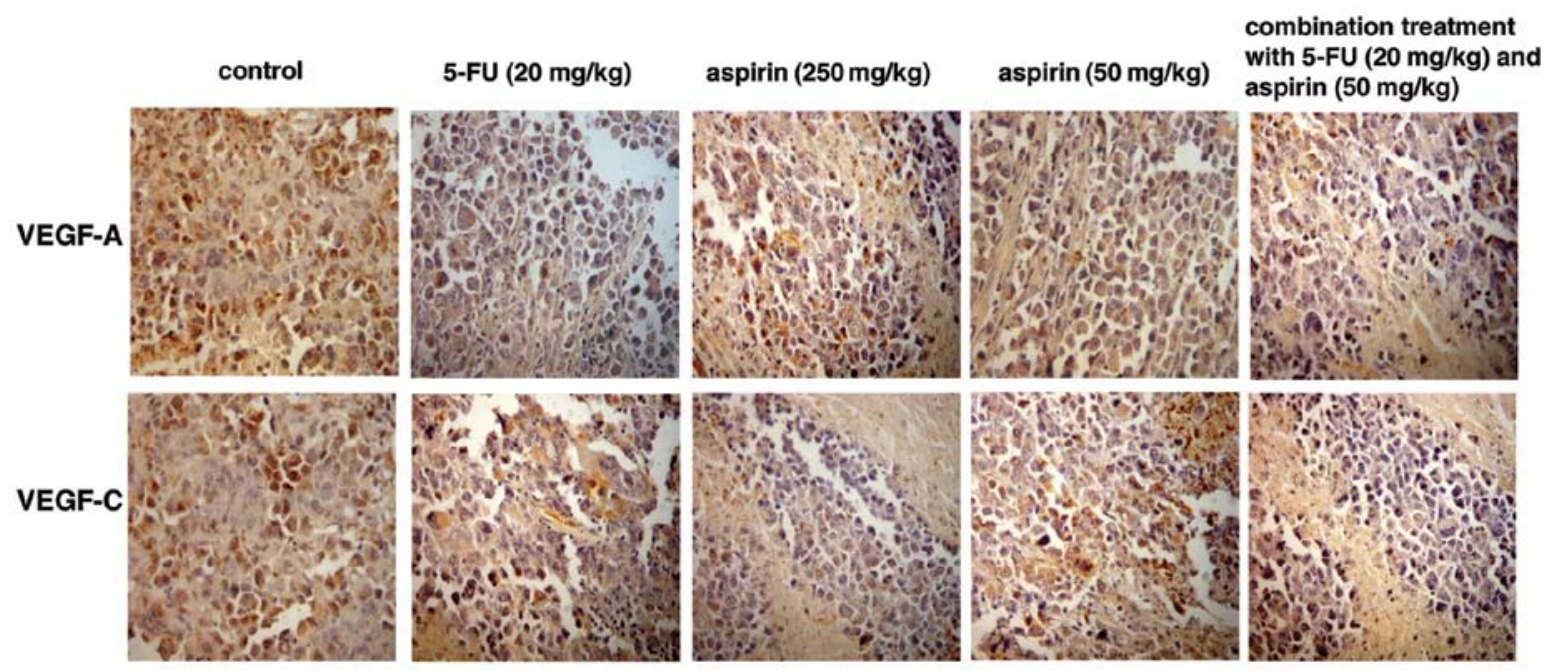

Figure 3. Effects of aspirin on the expression of VEGF-A and VEGF-C in S180 sarcoma tissue were detected by immunohistochemistry. Original magnification, $\mathrm{x} 400$.

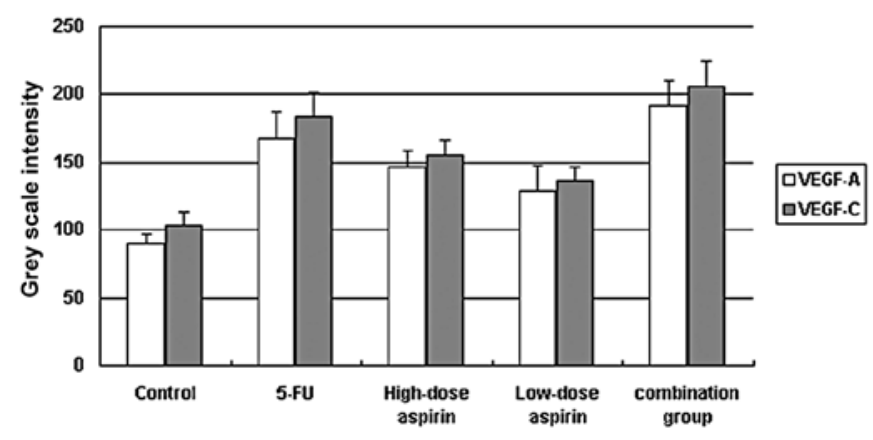

Figure 4. Grey scale intensity variants were evaluated by Leica Qwin V3 software for VEGF-A and VEGF-C expression in S180 sarcoma tissue. Sections in each of 5 randomly selected positive regions (original magnification $\mathrm{x} 200$ ). Higher grey scale intensity represents weaker protein expression, and lower, stronger protein expression. We found that expression of VEGF-A and VEGF-C protein decreased in S180 sarcoma tissues treated with 5-FU and aspirin particularly in the 5-FU alone and combination treatment groups compared to the control group. In each comparison, there was a significant difference $(\mathrm{P}<0.05)$.

Effect of aspirin treatment on VEGF-A and VEGF-C protein expression as assessed by western blot analysis. VEGF-A and VEGF-C expression was normalized to $\beta$-actin expression by
Table II. Counts of MVD and LVD in S180 sarcoma tissue (mean \pm SEM, $n=10)$.

\begin{tabular}{lcc}
\hline Group & MVD & LVD \\
\hline Control & $11.65 \pm 4.34$ & $6.45 \pm 2.03$ \\
5-FU & $7.13 \pm 2.16^{\mathrm{a}}$ & $5.20 \pm 1.61$ \\
High-dose aspirin & $7.55 \pm 2.73^{\mathrm{a}}$ & $4.63 \pm 1.84^{\mathrm{a}}$ \\
Low-dose aspirin & $9.87 \pm 3.89^{\mathrm{a}}$ & $5.47 \pm 1.34$ \\
Combination group & $6.82 \pm 1.30^{\mathrm{a}}$ & $4.95 \pm 1.03^{\mathrm{a}}$ \\
\hline
\end{tabular}

Control, treated with normal saline. ${ }^{\mathrm{a}} \mathrm{P}<0.05$ vs. the control.

band intensity. As known in Fig. 5, VEGF-A and VEGF-C expression was reduced in the high- and low-dose aspirin, 5-FU and combination groups. Band intensities were analyzed by ImageJ software. VEGF-A and VEGF-C expression decreased significantly in the S180 sarcoma tumors treated with 5-FU alone or with the combination with aspirin. The decreased expression also showed a dose-dependent trend in the high- and low-dose aspirin groups. 

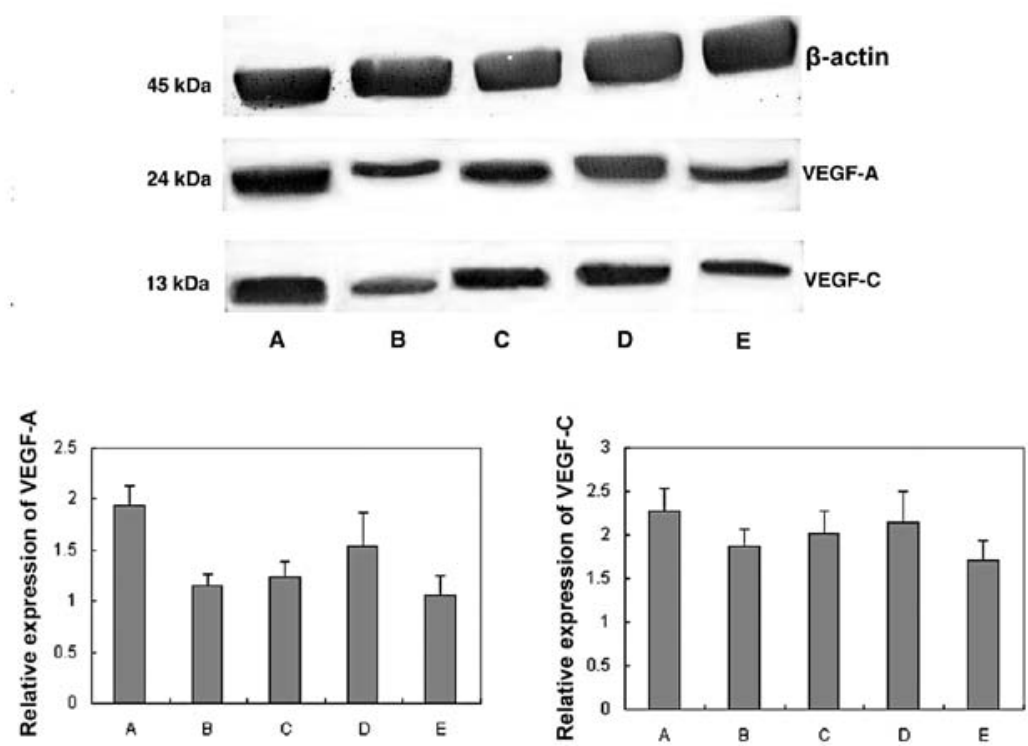

Figure 5. Effects of aspirin on expression of VEGF-A and VEGF-C in S180 sarcoma tissues were detected by western blot analysis. The relative expression of VEGF-A and VEGF-C was analyzed by ImageJ software. Columns in the histograms represent the mean of six separate experiments (intensity ratio of $\beta$-actin to VEGF-A or VEGF-C). Western blot analysis revealed that the $24 \mathrm{kDa}$ band of VEGF-A and the $13 \mathrm{kDa}$ band of VEGF-C protein in the 5-FU, aspirin and combination groups increased in intensity compared to the control group. In each comparison, a significant difference was achieved $(\mathrm{P}<0.05)$. A, control group; $\mathrm{B}$, 5-FU (20 mg/kg); C, aspirin (250 mg/kg); D, aspirin (50 mg/kg); E, combination treatment with 5-FU (20 mg/kg) and aspirin (50 mg/kg).

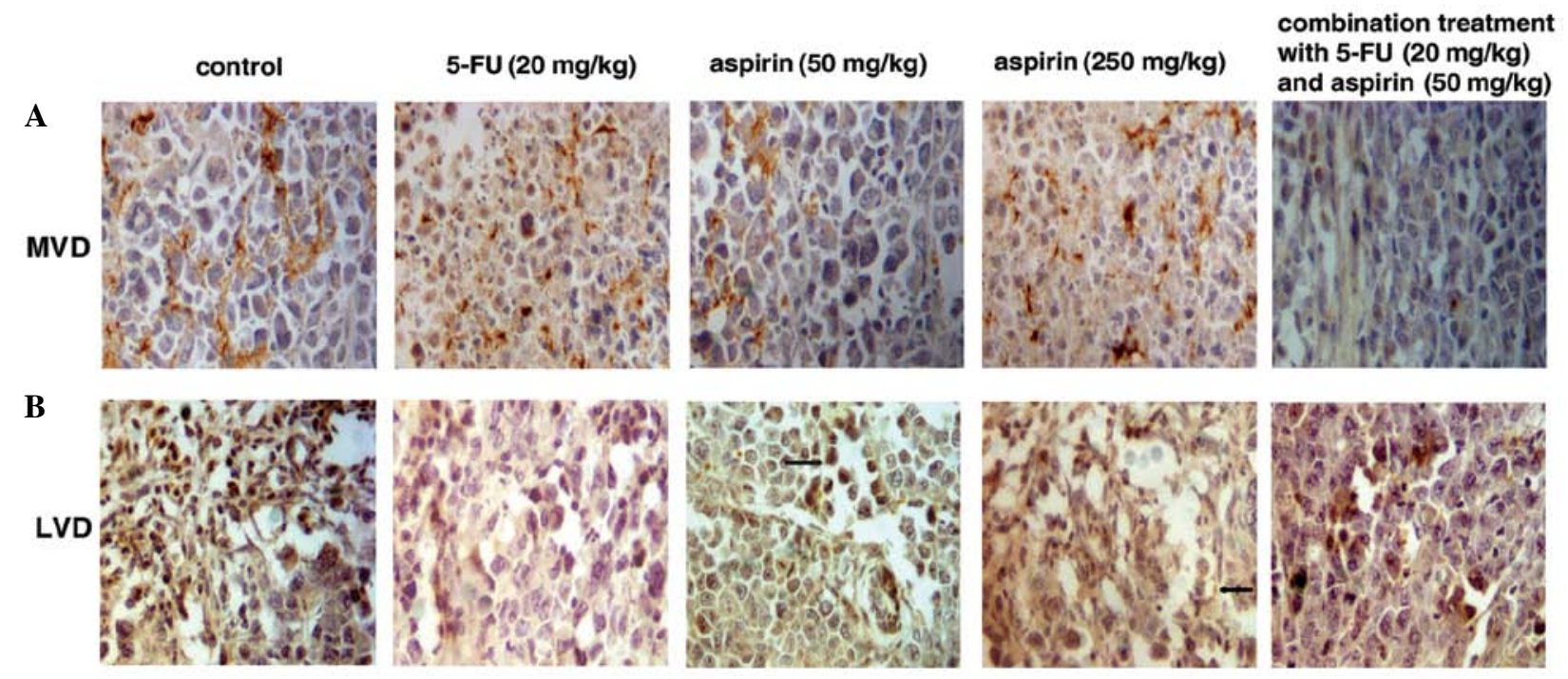

Figure 6. MVD and LVD were determined by immunohistochemical staining for CD34 and D2-40 in S180 sarcoma tissues, respectively. Original magnification, $\mathrm{x} 400$.

MVD and LVD in sarcoma 180 tumors. Cells positive for CD34 were stained brown. Microvessel distribution is shown in Fig. 6A. Necrotic tumor cells were frequently present in the 5-FU-treated group and in the high-dose aspirin group. The 5-FU alone, high- and low-dose aspirin and combination groups all demonstrated inhibition of MVD in comparison to the control group ( $\mathrm{P}<0.05$ for each comparison), which suggests that aspirin inhibits angiogenesis (Table II).

D2-40-positive tubular structures. D2-40-positive tubular structures were stained brown. The vessel walls were thin and irregular in structure (Fig. 6B). The 5-FU alone, aspirin and the combination groups had an inhibitory effect on LVD.
The high-dose aspirin $(250 \mathrm{mg} / \mathrm{kg})$ group showed a noticeable inhibition of LVD in comparison to the control group $(\mathrm{P}<0.05)$ (Table II). The high-dose aspirin group exhibited positive tumor cells generating non-endothelial cell-lined channels (Fig. 6B, arrow fourth panel). The low-dose aspirin group displayed a tumor cell around a lymphatic vessel (Fig. 6B, arrow, middle panel). The result revealed that LVD of the control group was the highest in the 5 groups and the lumen showed obvious expansion. The 5-FU, high- and low-dose aspirin groups had an inhibitory effect on LVD and VEGF-C. High-dose aspirin $(250 \mathrm{mg} / \mathrm{kg})$ markedly inhibited lymphatic vessel density when compared with the control group. 


\section{Discussion}

This research demonstrated the inhibitory effect of aspirin on murine S180 sarcoma growth in vivo and its possible mechanisms. The inhibitory rates of the 5 -FU, high-dose $(250 \mathrm{mg} / \mathrm{kg})$ and low-dose $(50 \mathrm{mg} / \mathrm{kg})$ aspirin, and combination of 5 -FU with aspirin groups were $64.1,33.5,22.2$ and $70.1 \%$, respectively. Unexpectedly, aspirin enhanced the antitumor effect of 5-FU, and the mice treated with aspirin were in good condition. In conclusion, aspirin is capable of reducing the body wasting caused by tumors.

Aspirin demonstrated a potential in decreasing the expression of VEGF-A and MVD. Tumor tissue in the highdose aspirin group showed patchy necrosis and decreased MVD. These results imply that antiangiogenesis plays a critical role in aspirin-induced antitumor activity. This is possibly due to the attributes of aspirin that directly affect vascular endothelial cells and decrease VEGF-A production along with MVD, thereby causing tumor tissue ischemia and even necrosis. Cox-2 is important in regulating angiogenesis, and its reported mode of action on endothelial cells is indirect (26). Our previous study showed that aspirin decreases the expression of Cox-2 in tumor tissue. Therefore, we concluded that aspirin inhibits angiogenesis by directly targeting endothelial cells through an independent Cox pathway. There are other possible pathways that could be responsible including pathways regulating ERK kinase, cyclin E and CDC2 (27). However, further research is needed to ascertain the pathway responsible for the inhibitory effect of aspirin on angiogenesis.

It is difficult to distinguish blood vessels from lymphatic vessels using regular H\&E staining. D2-40 was recently discovered to be a tumor-associated lymphatic vessel endothelial cell marker with high specificity (28). D2-40 was used to identify lymphatic endothelial cells in this research. The treatment groups had an inhibitory effect on LVD and VEGF-C particularly in the high-dose aspirin group $(250 \mathrm{mg} / \mathrm{kg})$. We concluded that aspirin inhibits tumor lymphangiogenesis by reducing VEGF-C consequently inhibiting the growth of murine S180 sarcoma. Moreover, we found that positive tumor cells generated non-endothelial cell-lined channels (arrow in Fig. 6B), which indicated that fast growing tumors require various nutrients and need additional channels in order to transport nutrients. A tumor cell around a lymphatic vessel (arrow in Fig. 6B) was noted, which indicated that the tumor cell might spread through the lymphatic vessel. Several studies had suggested that the level of Cox-2 expression in tumor tissue is correlated with lymph node metastasis (21-23), and Cox-2 promotes tumor lymphangiogenesis and consequent lymph node metastasis (29). We indicated here, that aspirin inhibits tumor lymphangiogenesis through VEGF-C dependent on the reduced activity of Cox-2.

A recent study showed that daily usage of low-dose aspirin $(75-300 \mathrm{mg}$ ) can reduce the incidence of cancer by $\sim 25 \%$ after a delay of $\sim 3$ years. This provides strong evidence supporting our conclusion, but it also suggests that the side effects of aspirin such as anemia and bleeding might account for the subsequent reduction in cancer death (4). In our study, tumor-bearing mice treated with aspirin had slight to mild side effects. This was possibly due to the short treatment duration. Aspirin is indeed safer than most chemotherapeutic agents; however, we still need to optimize the dose for cancer treatment.

In summary, our findings demonstrated that aspirin can inhibit tumor angiogenesis by reducing the production of VEGF-A, and inhibiting tumor lymphangiogenesis through the reduction of VEGF-C in a dose-dependent manner, which explains why aspirin inhibits the tumor growth of murine S180 sarcoma in vivo. We also found that aspirin synergistically enhanced the antitumor effect of 5-FU. In addition, the significance of these finding needs to be established in a broader context by conducting further studies in a variety of tumor models. To the best of our knowledge, our findings indicate that aspirin and other NSAIDs inhibit lymphangiogenesis, possibly through COX-2-dependent regulation of VEGF-C expression. Although further validation is required, the proposed effect of aspirin specifically on tumor angiogenesis and lymphangiogenesis may have therapeutic implications in chemoprevention, adjuvant chemotherapy and treatment of metastatic disease.

\section{Acknowledgements}

This study was supported by funding from the National Nature Science Foundation of China (nos. 81073102 and 30873408) and Development Project of Science and Technology in Jinan (no. 201004012).

\section{References}

1. Vinogradova Y, Hippisley-Cox J, Coupland C, et al: Risk of colorectal cancer in patients prescribed statins, nonsteroidal antiinflammatory drugs, and cyclooxygenase-2 inhibitors: nested case-control study. Gastroenterology 133: 393-402, 2007.

2. Doherty GA and Murray FE: Cyclooxygenase as a target for chemoprevention in colorectal cancer: lost cause or a concept coming of age? Expert Opin Ther Targets 13: 209-218, 2009.

3. Rostom A, Dube C, Lewin G, et al: Nonsteroidal anti-inflammatory drugs and cyclooxygenase- 2 inhibitors for primary prevention of colorectal cancer: a systematic review prepared for the U.S. Preventive Services Task Force. Ann Intern Med 146: 376-389, 2007.

4. Rothwell PM, Price JF, Fowkes FG, et al: Short-term effects of daily aspirin on cancer incidence, mortality, and non-vascular death: analysis of the time course of risk sand benefits in 51 randomised controlled trials. Lancet 379: 1602-1612, 2012.

5. Li S, Miner K, Fannin R, et al: Cyclooxygenase-1 and 2 in normal and malignant human ovarian epithelium. Gynecol Oncol 92: 622-627, 2004

6. Thun MJ, Henley SJ and Patrono C: Nonsteroidal anti-inflammatory drugs as anticancer agents: mechanistic, pharmacologic, and clinical issues. J Natl Cancer Inst 94: 252-266, 2002.

7. Dube C, Rostom A, Lewin G, et al: The use of aspirin for primary prevention of colorectal cancer: a systematic review prepared for the U.S. Preventive Services Task Force. Ann Intern Med 146: 365-375, 2007.

8. Sadeghi S, Bain CJ, Pandeya N, et al: Aspirin, nonsteroidal antiinflammatory drugs, and the risks of cancers of the esophagus. Cancer Epidemiol Biomarkers Prev 17: 1169-1178, 2008.

9. Gee JR, Jarrard DF, Bruskewitz RC, et al: Reduced bladder cancer recurrence rate with cardioprotective aspirin after intravesical bacille Calmette-Guerin. BJU Int 103: 736-739, 2009.

10. Kelly JP, Coogan P, Strom BL, et al: Lung cancer and regular use of aspirin and nonaspirin nonsteroidal anti-inflammatory drugs. Pharmacoepidemiol Drug Saf 17: 322-327, 2008.

11. Alfonso LF, Srivenugopal KS, Arumugam TV, et al: Aspirin inhibits camptothecin-induced $21^{\mathrm{CIP} 1}$ levels and potentiates apoptosis in human breast cancer cells. Int J Oncol 34: 597-608, 2009. 
12. Kim SR, Bae MK, Kim JY, et al: Aspirin induces apoptosis through the blockade of IL-6-STAT3 signaling pathway in human glioblastoma A172 cells. Biochem Biophys Res Communm 387: 342-347, 2009

13. Iwama T: NSAIDs and colorectal cancer prevention. J Gastroenterol 44: 72-76, 2009.

14. Dannenberg AJ, Altorki NK, Boyle JO, et al: Cyclo-oxygenase 2: a pharmacological target for the prevention of cancer. Lancet Oncol 2: 544-551, 2001 .

15. Wang D, Mann JR and DuBois RN: The role of prostaglandins and other eicosanoids in the gastrointestinal tract. Gastroenterology 128: 1445-1461, 2005.

16. Alitalo K, Tammela T and Petrova TV: Lymphangiogenesis in development and human disease. Nature 438: 946-953, 2005.

17. Achen MG and Stacker SA: Tumor lymphagiogenesis and metastatic spread-new players begin to emerge. Int J Cancer 119 1755-1760, 2006

18. Pepper MS, Tille JC, Nisato R, et al: Lymphangiogenesis and tumor metastasis. Cell Tissue Res 314: 167-177, 2003.

19. Yonemura Y, Endo Y, Fujita H, et al: Role of vascular endothelial growth factor $\mathrm{C}$ expression in the development of lymph node metastasis in gastric cancer. Clin Cancer Res 5: 1823-1829, 1999.

20. Su JL, Shih JY, Yen ML, et al: Cyclooxygenase-2 induces EP1and HER-2/Neu-dependent vascular endothelial growth factor-C up-regulation: a novel mechanism of lymphangiogenesis in lung adenocarcinoma. Cancer Res 64: 554-564, 2004

21. Timoshenko AV, Chakraborty C, Wagner GF, et al: COX-2mediated stimulation of the lymphangiogenic factor VEGF-C in human breast cancer. Br J Cancer 94: 1154-1163, 2006.
22. Siironen P, Ristimaki A, Narko K, et al: VEGF-C and COX-2 expression in papillary thyroid cancer. Endocr Relat Cancer 13: 465-473, 2006

23. Soumaoro LT, Uetake H, Takagi Y, et al: Coexpression of VEGF-C and Cox-2 in human colorectal cancer and its association with lymph node metastasis. Dis Colon Rectum 49: 392-398, 2006.

24. Zhang J, Ji J, Yuan F, et al: Cyclooxygenase-2 expression is associated with VEGF-C and lymph node metastases in gastric cancer patients. Biomed Pharmacother 59: S285-S288, 2005.

25. Weider N, Carroll PR, Flax J, et al: Tumor angiogenesis correlation with metastasis in invasive prostate carcinoma. Am J Pathol 143: 755-761, 1993.

26. Borthwick GM, Johnson AS, Partington M, et al: Therapeutic levels of aspirin and salicylate directly inhibit a model of angiogenesis through a Cox-independent mechanism. FASEB J 20: 2009-2016, 2006.

27. Pillinger MH, Capodici C, Rosenthal $\mathrm{P}$, et al: Modes of action of aspirin-like drugs: salicylates inhibit erk activation and integrindependent neutrophil adhesion. Proc Natl Acad Sci USA 95: 14540-14545, 1998.

28. Saad RS, Kordunsky L, Liu YL, et al: Lymphatic microvessel density as prognostic marker in colorectal cancer. Mod Pathol 19: $1317-1323,2006$

29. lwata C, Kano MR, Komuro A, et al: Inhibition of cyclooxygenase-2 suppresses lymph node metastasis via reduction of lymphangiogenesis. Cancer Res 67: 10181-10189, 2007. 